\title{
The Effect of Visible Light on the Gunn Rat: Convulsive Threshold, Bilirubin Concentration, and Brain Color
}

\author{
Thomas R. G. Sisson, ${ }^{[12]}$ Steven Goldberg, and Barry Slaven \\ Department of Pediatrics, Temple University School of Medicine and St. Christopher's Hospital for Children, \\ Philadelphia, Pennsylvania, USA
}

\begin{abstract}
Extract
Irradiation of congenitally jaundiced rats from the 1st-21st days of life by visible light emitting between 420 and $470 \mathrm{~nm}$ is effective in preventing hyperbilirubinemia, brain discoloration by bilirubin, and ataxia, in maintaining normal cerebellar size, and in preserving normal convulsive threshold. These findings indicate the prevention of bilirubin encephalopathy. Irradiation by "daylight" fluorescent light was only partially effective. The results of this study also indicate that in vivo photodecomposition of bilirubin has a dose-response relation to the emission in the absorbance range of the pigment.
\end{abstract}

\section{Speculation}

The prevention of bilirubin encephalopathy in human neonates during phototherapy may be enhanced by the direct action of visible light on bilirubin circulating in the vessels of the brain. The transmission of light into this organ would be a fruitful area of study to confirm or discard this hypothesis. Measurement of the convulsive threshold of suitable animals may offer a quantified basis of comparison of various phototherapeutic regimens in the study of hyperbilirubinemia.

\section{Introduction}

Photodecomposition of bilirubin in vivo may be effected by irradiation of the body by visible light. Fluorescent lamps of broad spectral output ("daylight" and "blue") and lamps with narrow spectral emission ("special blue" F20T12/BB [10]) are all used in phototherapy of hyperbilirubinemia in the neonate for the purpose of preventing bilirubin encephalopathy. Under banks of these lamps serum bilirubin concentrations are reduced by photooxidative processes (and perhaps by accelerated bilirubin excretion in the bile) with varying degrees of efficiency and length of exposure [7].
We lack certain knowledge that phototherapy exerts a real effect in reducing the incidence of neurologic sequelae of neonatal jaundice, especially among infants of low birth weight, although the long term studies of Lucey et al. [5] offer reassurance on this point, and at this institution gross evidence of brain damage has not been encountered in initial studies at 12 months of age in infants so treated during the neonatal period.

\section{Materials and Methods}

This study was undertaken to determine whether prevention of brain damage from bilirubin toxicity by the 
use of phototherapy could be shown by other means. Because such an investigation could not be carried out in the human, a special strain of hooded Gunn rat was selected as an animal model. This strain is characterized by a congenital deficiency of glucuronyltransferase. The homozygous rats of this type are congenitally icteric and develop bilirubin encephalopathy with marked neurologic damage manifested by ataxia, wide stance, spasticity, low held rump, shortened life span, and deep orange staining of brain tissue. Heterozygotes exhibit neither these signs and symptoms nor hyperbilirubinemia.

It has been demonstrated [1] that the convulsive threshold of rats may be affected by brain damage and some neurotropic drugs, and that this may be measured by the response to convulsive agents. A comparison of the convulsive thresholds of normal, nonjaundiced heterozygous Gunn rats, jaundiced homozygous Gunn rats, and light-irradiated rats of both types was made in this study. The time of onset of convulsions, induced by inhalation of bis-2,2,2-triffuorethyl ether (Indaklon) in a 10\% alcohol solution, was measured to indicate the presence or absence of brain damage by hyperbilirubinemia, and the possible protective effect of light irradiation in the naturally icteric strain. Shortening of the convulsive threshold in point of time between infusion of the convulsant and the onset of clonic seizures was taken to indicate abnormal brain function, damage caused by bilirubin toxicity.

The 208 rats in the study were divided into three groups, into which were placed jaundiced and nonjaundiced members of different litters so that interlitter variables would be eliminated. Maximum litter size was 10 pups. Each group was maintained for 21 days in cages with nursing mothers to ensure adequate diet and fluid intake. The environmental lighting, described below, was constant. On the 22 nd day of life, rats were placed individually in a closed 1-gallon jar into which was infused the volatile convulsant ether at a rate of $0.103 \mathrm{ml} / \mathrm{min}$, and the time of onset of clonic convulsions measured by stopwatch.

Group $A$ consisted of 69 rats raised from birth in the dark. They served as controls. Of these, 38 were jaundiced homozygotes (jj), and 31 were nonjaundiced heterozygotes $(\mathrm{jJ})$.

Group B consisted of 55 rats raised from birth under broad spectrum daylight fluorescent lamps (F20T12/D), whose energy output or flux, at $400-490 \mathrm{~nm}$, was 0.09 $\mathrm{mW} / \mathrm{cm}^{2}$. Of these rats, 24 were jaundiced and 31 were nonjaundiced.

Group $C$ consisted of 84 rats raised from birth under special blue fluorescent lamps (F20T12/BB) of narrow spectral radiance $(420-470 \mathrm{~nm})$ whose principal energy output was at $445 \mathrm{~nm}$, with a total flux of $0.33 \mathrm{~mW} / \mathrm{cm}^{2}$. Of these rats, 35 were jaundiced and 49 were not.

The age of 21-22 days for testing was chosen because preliminary studies in this laboratory demonstrated, in 60 rats convulsed at 12, 15, 17, 19, and 21 days of age, that the response to the convulsant is erratic and difficult to interpret before 21 days, presumably because of developmental neuronal immaturity.

Serum bilirubin concentration of each rat was determined in duplicate samples in an AO Bilirubinometer [11] from blood drawn shortly after recovery from the induced seizure. On duplicate aliquots, concentrations were measured in 60 rats by the spectrophotometric method of White et al. [9] to assess the accuracy of the Bilirubinometer. No variation of more than $3 \%$ was encountered.

\section{Results}

The mean convulsion times and serum bilirubin concentrations of the rats at 21-22 days of age are recorded in Table I.

The nonjaundiced heterozygotes in each group had a mean threshold of about $500 \mathrm{sec}$. The lighting environment did not alter this. The jaundiced rats raised in the dark (group $A)$, and those raised under daylight fluorescent lamps (group $B$ ) had a significantly shorter mean convulsion time, 345 and $371 \mathrm{sec}$, respectively, than the rats raised under narrow spectrum blue light (group $C$ ), whose mean time was $508 \mathrm{sec}$, equal to the heterozygous controls.

The serum bilirubin concentrations of the heterozygous rats in all three groups were low (mean $=0.06-$ $0.7 \mathrm{mg} / 100 \mathrm{ml}$ ). Only the homozygous rats of group $C$ had low values of serum bilirubin (mean $=4.3 \mathrm{mg}$ / $100 \mathrm{ml}$ ), which demonstrated that the pure blue light radiation was effective in maintaining the bilirubin concentrations at significantly reduced levels. The homozygous rats of group $B$ had a wide range of serum bilirubin concentrations (mean $=10.8 \pm 5.7$ $\mathrm{mg} / 100 \mathrm{mI}$ ), which indicated that the lower flux in the blue range from the daylight lamps compared with that of the narrow spectrum blue lamps was less effective in reducing serum bilirubin and maintaining low levels of concentration.

Inspection of the brains of heterozygous nonicteric rats in each group revealed that they were of a uni- 
Table I. Convulsion times and bilirubin concentrations of control and trcated Gunn rats, with comparison of all groups by chi-square analysis

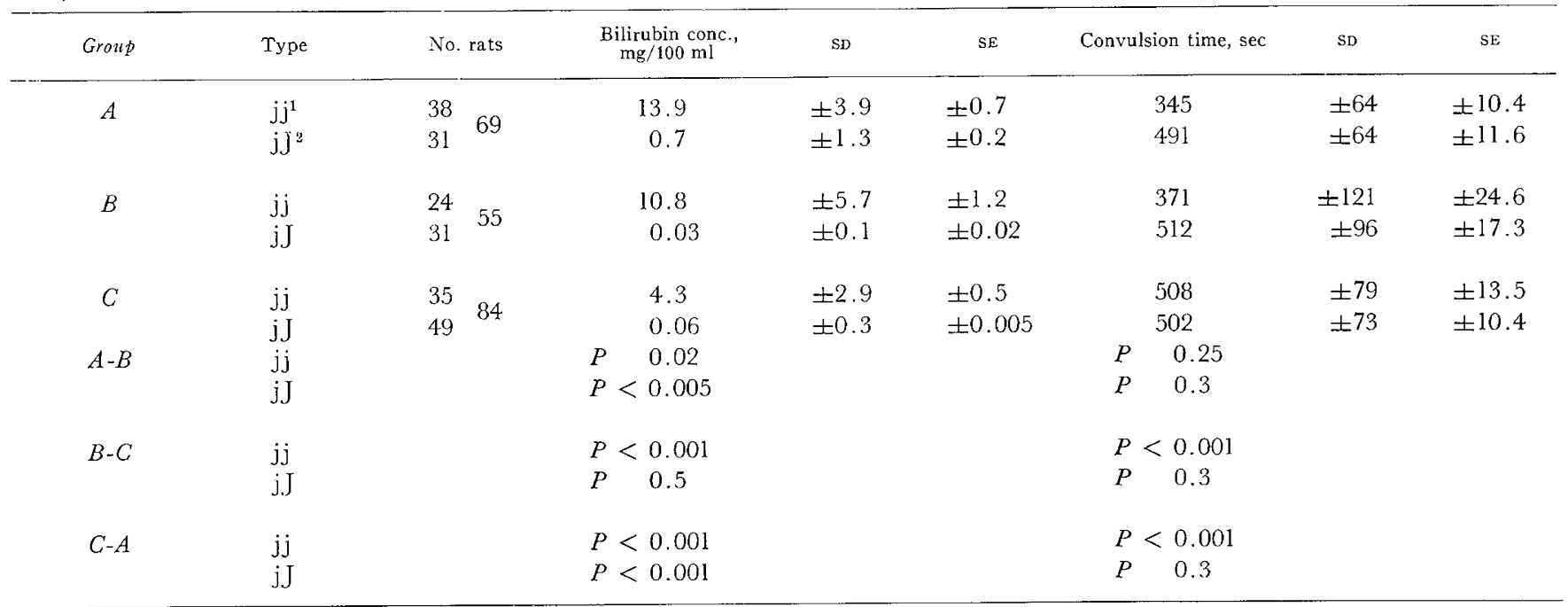

$1 \mathrm{jj}$ : jaundiced homozygote.

$2 \mathrm{jJ}$ : nonjaundiced heterozygote.

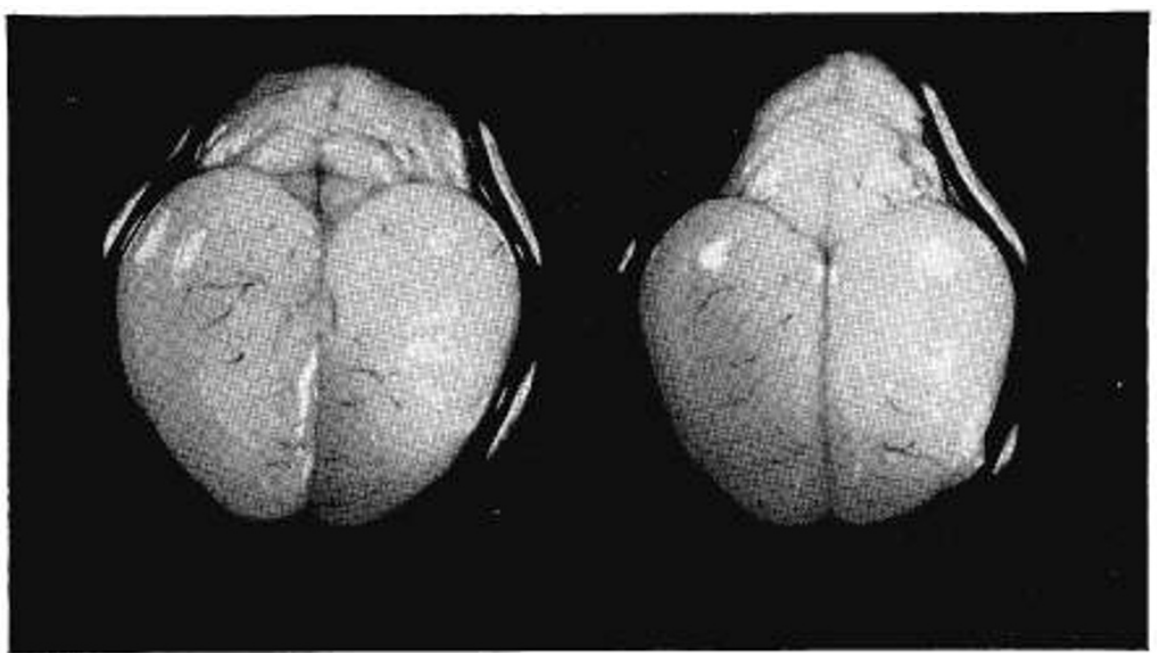

Fig. 1. The brains of two Gunn rats, aged 21 days. Both rats were homozygous for congenital icterus (glucuronyltransferase deficiency). The brain on the left, heavily stained a yellow-orange, is from a rat raised in the dark. Note the small cerebellum. The brain on the right is from a rat of the same age raistd under blue fluorescent light. The tissue is normal in color, and the cerebellum is of normal size.

form pearly beige color. The brains of homozygotes in group $A$, raised in the dark, were intensely yelloworange in hue. The homozygotes of group $B$ had brains with moderate yellow staining in about $50 \%$ of instances, coincidental with elevation of serum bilirubin, the rest exhibiting the beige of controls. In homozygotes of group $C$ the brains were all of the beige of controls.

It may be observed in Figure 1 that the cerebellum of the brain from an unirradiated homozygous rat of group $A$ is appreciably smaller than that of the other brain, which was removed from an irradiated homozygote of group $C$. This disparity in cerebellar size was observed between all the homozygotes of group $A$ and the irradiated homozygotes of groups $B$ and $C$, as well as the heterozygotes of all groups. The cerebella of group $C$ homozygotes were almost uniformly the size of heterozygous controls, and the group $B$ homozygotes had this uniformity in about $70 \%$ of instances.

Johnson and Schutta [3] have shown that brain cells of jaundiced Gunn rats, when damaged by bilirubin, become necrotized and disintegrate, eventually disap- 
Table II. Body weight of Gunn rats at 21 days of age

\begin{tabular}{cccccc}
\hline & \multicolumn{5}{c}{ Genotype } \\
\cline { 2 - 4 } \cline { 5 - 6 } \cline { 5 - 6 } & \multicolumn{3}{c}{$\mathrm{jj}$} & $\mathrm{g}$ & $\mathrm{jJ}$ \\
\cline { 2 - 6 } & $\mathrm{g}$ & $\mathrm{sD}$ & & $\mathrm{g}$ & \\
\hline$A$ & 35.5 & \pm 6.4 & & 41.1 & \pm 5.9 \\
$B$ & 33.1 & \pm 6.6 & & 41.0 & \pm 6.0 \\
$C$ & 36.8 & \pm 10.0 & & 40.7 & \pm 6.4 \\
\hline
\end{tabular}

pearing. The total organ size is thus affected, notably the cerebellum, and we believe that this explains in part the smaller cerebellar size of the homozygotes in group $A$ and of those in group $B$ whose bilirubin concentrations were elevated.

A statistical comparison of mean convulsion times versus mean bilirubin concentrations for each group shows that the convulsion times vary inversely with the concentration of bilirubin. Analysis by $t$ test gave a correlation coefficient of $-0.95, P<0.005$, which is an undoubtedly significant relation.

The bilirubin concentrations of the nonjaundiced rats of the three groups hardly differed. There was a significant difference, however, between the jaundiced rats of groups $B$ and $C(P<0.001)$, but only a minor difference was shown between the jaundiced rats of groups $A$ and $B$ ( $P$ 0.02). These data indicate that the narrow spectrum blue lamp irradiation was more effective in reducing the bilirubin concentration, whereas the broad spectrum daylight radiation was less effective in reducing the bilirubin concentrations in that group but little different from the dark control levels.

Similar statistical analysis of the data concerned with convulsion times revealed highly significant differences between the jaundiced and nonjaundiced rats of group $A(P<0.001)$ and of group $B(P<0.001)$, but not of group $C(P 0.7)$. This indicates that only the narrow spectrum blue light irradiation prevented a shortened convulsion time in the homozygous rats. A comparison of the convulsion times of dark control homozygotes in group $A$ with like rats in group $B$ treated with daylight lamps showed no difference $(P$ $0.25)$, whereas a comparison of this type in groups $B$ and $C$ showed a very significant difference $(P<0.001)$.

The mean body weights of the three groups of rats on the 21st day of life are shown in Table II. Characteristically, the jaundiced homozygotes are somewhat smaller than the unaffected heterozygotes. There was $n n$ significant difference in the weights of either type of rat ( $\mathrm{jj}$ or $\mathrm{jJ}$ ), whether raised in the dark or constantly exposed to broad or narrow spectrum blue light (group $A$ versus $B, P 0.15 ; B$ versus $C, P 0.1 ; A$ versus $C, P 0.45$ ). These data support the conclusions of a previous study [4], that constant exposure to light does not retard the growth rate of Gunn rats.

\section{Discussion}

It has been demonstrated $[2,6]$ that the most effective range of visible light for the photodestruction of bilirubin in vitro is in the blue portion of the spectrum, which corresponds to the wavelengths absorbed by the pigment and some breakdown products. It has also been shown [7] that, in its clinical application for phototherapy of neonatal jaundice, blue light is more efficient in respect to rate of serum bilirubin decrease and length of exposure time than broad spectrum or white light, whose blue component is only a fraction of the total radiant intensity of the light source rather than the sole or major component. The results of this investigation support these previous studies, giving further evidence that there is a dose-response relation of bilirubin photodestruction to energy output of visible light in the wavelengths of the action spectrum of the pigment.

It may be seen in Table I that the dark control jaundiced rats, and those rats raised under broad spectrum lamps, had a similar shortened convulsion time, whereas a normal convulsion time was demonstrated in the jaundiced rats raised under narrow spectrum radiation. This indicates that the prevention of brain damage was not achieved reliably by the light source of lesser energy in the blue.

Although the light exposure times were equal, day light fluorescents did not uniformly prevent staining or yellow discoloration of the brains as did the pure blue sources. It is possible that the lack of staining in the rats of group $C$ may have been due only in part to the maintenance of low serum bilirubin concentrations, for Viggiani et al. [8] have reported that a significant amount of light can penetrate the skin and skull of the rat, directly impinging upon and penetrating the brain substance.

The conclusion that bilirubin encephalopathy in rats is minimized, if not wholly prevented, by adequate light irradiation does not prove that the same result can be attained by phototherapy of hyperbilirubinemia in the human infant. The presumption that it may do so is strongly implied by this study, but proof must await more long term evaluation of infants treated for this condition.

The choice of lamp sources for phototherapy still 
rests on personal conviction, on advertising claims, and to some degree on trial and error, for the optimum radiant energy has not been demonstrated. It is clear that broad spectrum lamp emission, if it is energetic enough, should be, and is, effective in the photodegradation of bilirubin. However, the total levels of radiation must be relatively high to attain this end, and such energies in other wavelengths than blue may have undesirable effects not related to bilirubin breakdown. The use of a narrow spectrum lamp appears to be more efficient in this singular photochemical reaction, but little more is known of its other possible effects than of broad spectrum light. Before unconditional recommendation of one or the other source can be made, more complete study of their photobiologic activities is necessary.

\section{Summary}

These studies indicate that irradiation of congenitally jaundiced Gunn rats by visible light emitting between 420 and $470 \mathrm{~nm}$ preserves the normal convulsive threshold so that it is equal to that of nonjaundiced control rats, and that irradiation by daylight fluorescent light is significantly less effective. Yellow pigmentation of the brains, and small cerebellar size was not uniformly inhibited by the daylight lamps, in contrast to the lack of pigmentation and normal cerebella observed in rats raised under a pure blue light. The homozygous animals raised in the dark were ataxic, demonstrating gross brain damage, whereas those exposed to blue light were not. Some of the homozygotes raised under daylight lamps exhibited ataxia and wide stance, indicating some degree of neurologic injury.

These findings led us to conclude that irradiation by daylight fluorescent lamps is only partially effective in preventing jaundice in these animals, in preventing brain damage from bilirubin toxicity, as demonstrated by the low convulsive threshold. On the other hand, irradiation by a pure blue fluorescent light source, lower in footcandles but higher in energy output in the narrow range of $420-470 \mathrm{~nm}$, was effective in preventing hyperbilirubinemia, brain discoloration, and ataxia, in maintaining normal cerebellar size, and in preserving a normal convulsive threshold.

These data further indicate that in vivo photodegradation of bilirubin has a dose-response relation to the light source, which source must contain an emission in the absorbance range of bilirubin to be effective.

\section{References and Notes}

1. Adler, M. W., Sagel, S., Kittagawa, T. S., and Maynert, E. W.: The effects of repeated fluorethyl-induced seizures on convulsive threshold and brain monoamines in rats. Arch. Int. Pharmacodyn., 170: 12 (1967).

2. Blondheim, S. H., Lathrop, D., and Zabirskie, J.: Effect of light on absorption spectrum of jaundiced serum. J. Lab. Clin. Med., 60: 31 (1962).

3. Johnson, L., ANd Schutta, H. S.: Bilirubin encephalopathy in the Gunn rat: Fine structure study of the cerebellar cortex. J. Neuropathol. Exp. Neurol., 26: 377 (1967).

4. Kendall, S., Goldberg, S., and Sisson, T. R. C.: Influence of visible light on the growth rates of Gunn rats (Abstract). Clin. Res., 18: 692 (1970).

5. Lucey, J. F., Hewitt, J. R., Emery, E. S., Goldstein, S., AND Colcrins, S.: Controlled follow-up study of low birth weight infants at 4-6 years of age treated with phototherapy (Abstract). Pediat. Res., 7: 313 (1973).

6. Sisson, T. R. C., Kendall, N., Davies, R. E., And Berger, D.: Factors influencing the effectiveness of phototherapy in neonatal hyperbilirubinemia. Birth Defects: Original Article Series. 6: 100 (1970).

7. Sisson, T. R. C., Kendall, N., Shaw, E., and KechavarzOliaI, L.: Phototherapy of jaundice in the newborn. II. Effect of various light intensitites. J. Pediat., 81: 35 (1972).

8. Viggiani, E., Ciesla, M., and Russo, O. L.: Penetrazione della luce attraverso il cranio ed il cervello del ratte a diverse eta. Misurazioni eseguitese paratamente per la cute e per la parte ossea. Boll. Soc. Ital. Biol. Speriment., 46: 470 (1970).

9. White, D., Haidar, G. A., And Reinhold, J. G.: Spectrophotometric measurement of bilirubin concentration in the serum of the newborn by use of a microcapillary method. Clin. Chem., 4: 211 (1958).

10. Manufactured and supplied by Westinghouse Corporation, Bloomfield, N. J., courtesy of Dr. J. W. Sausville.

11. American Optical Company, Buffalo, N.Y.

12. Requests for reprints should be addressed to: T. R. C. Srsson, M.D., Department of Pediatrics, Temple University School of Medicine, 3400 N. Broad St., Philadelphia, Penna. 19140 (USA).

13. Accepted for publication January 29, 1974. 